\title{
CUESTIONES DEL DESARROLLO POLÍTICO: LAS FRÁGILES DEMOCRACIAS LATINOAMERICANAS
}

Armando Albuquerque

Doctor en Ciencia Política por la Universidad Federal de Pernambuco (UFPE/Brasil). Docente Titular del Programa de Postgrado en Derecho del Centro Universitario de João Pessoa-PB (PPGD/UNIPE). Docente colaborador del Programa de Postgrado en Ciencias Jurídicas de la Universidad Federal de Paraíba (PPGCJ/ UFPB). Líder del Grupo de Investigación "Instituciones de la Democracia, del Estado de Derecho y de la Ciudadanía en Brasil y América Latina"- PPGCJ/UFPB/ CNPq.

\section{Maria Aurea BaroniCecato}

Doctora en Derecho del Trabajo por la Universidad de Paris II - Panthéon-Assas (U-II - Francia). Docente Titular y Coordinadora del Programa de Postgrado en Derecho del Centro Universitario de João Pessoa-PB (PPGD/UNIPE). Docente colaborador del Programa de Postgrado en Ciencias Jurídicas de la Universidad Federal de Paraíba (PPGCJ/UFPB). Líderdel Grupo de Investigación "Trabajo y Desarrollo: Influjos y Disensiones" PPGCJ/UFPB/CNPq.

\section{Resumen}

El desarrollo político, como campo de investigación, tuvo sus orígenes en la segunda mitad del siglo pasado. Desde los años 60, cuando empezó a florecer, pasó a constituirse una frontera conceptual con la política comparada. En las décadas siguientes hubo muchos avances en el análisis de problemas relacionados con las instituciones políticas, las estructuras estatales, la sociedad civil, la estabilidad y crisis de los regímenes democráticos, entre otros. A partir del tema, se buscará en este trabajo, tomando como base el Democracy Index, analizar las causas de una de las principales deficiencias del desarrollo político en América Latina, a saber, la mala calidad de sus democracias y su no consolidación.

\section{Palabras clave}

Desarrollo político; Democracia; América Latina.

\section{Abstract}

The political development, as research field, had its origins in the second half of the last century. From the 60s, when he began to flourish, now constitutes a conceptual 
border with comparative politics. In subsequent decades there were many advances in the analysis of problems related to the political institutions, state structures, the rule of law, civil society, stability and crises of democratic regimes, among others. From this issue, will be made to this article, supported by the Democracy Index, analyzing the causes of one of the main shortcomings of the political development in Latin America, namely, the low quality of their democracies and the consequent non-consolidation of the same.

\section{Key words}

Crisis of Democratic Regimes; Political Institutions; Failures of the Political Development.

\section{Introducción}

El desarrollo político, como campo de investigación, tuvo sus orígenes en la segunda mitad del siglo pasado. Desde los años 60, cuando empezó a florecer, pasó a constituirse una frontera conceptual con la política comparada ${ }^{1}$. En las décadas siguientes hubo muchos avances en el análisis de problemas relacionados con las instituciones políticas, las estructuras estatales, la sociedad civil, la estabilidad y crisis de los regímenes democráticos, entre otros.

A partir del tema, se buscará en este trabajo, tomando como base el Democracy Index do Economist Intelligence Unit, analizar las causas de una de las principales deficiencias del desarrollo político en América Latina, a saber, la mala calidad de sus democracias y su no consolidación. Así que, la primera premisa se basa en que la mayor parte de las democracias latinoamericanas es de mala calidad y por lo tanto no consolidada.

La propagación mundial de la democracia fue uno de los principales acontecimientos políticos del último cuarto del siglo XX. Huntington (1991) llamó a esta expansión democrática de la tercera ola ${ }^{2}$. Antes de esa ola, el número de democracias en todos los continentes era aproximadamente 40. Al final de 1995 podrían encontrarse entre 76 y 117 democracias repartidas entre los continentes. La diferencia entre estos números es consecuencia de la concepción del régimen político. (Diamond 1999).

1 Cf. Hagopian (2000).

2 La expansión de la democracia comienza en el sur de Europa a mediados de los años 1970 y llega a América Latina a finales de esa década, ya principios de los ańos 1980. Llega al este, sur y sureste de Asia en la segunda mitad de la década de 80. El año 1989 estuvo marcado por la caída apoteótica del muro de Berlín, presagiando el fin del comunismo, de la reunificación alemana y el colapso de la Unión Soviética. En el Caribe esta tendencia también ocurre en los años 1990. Finalmente, en esta misma década la democracia llega a Sudáfrica. 
La ola de democratización comenzó en América Latina en 1978 y fue acompañada por avances y retrocesos ${ }^{3}$. Esto se debe, en cierta medida, a la forma como que se produjo su liberalización y procesos de transición. Tales procesos fueron, en la mayor parte de los casos, incompletos. Así, la segunda premisa aquí planteada es que la mala calidad de las democracias de América Latina y su no consolidación se deben, en cierta medida, a un proceso inacabado de democratización, lo que favoreció el aspecto electoral en detrimento del aspecto liberal de este régimen de gobierno.

Por tratarse de un artículo, no se tiene la intención de llevar a cabo una discusión teórica sobre el desarrollo político ${ }^{4}$, ni de la teoría democrática contemporánea ${ }^{5}$. En este sentido, se hace es necesario tomar dos conceptuaciones centrales para lograr el intento que aquí se propone.

La primera se refiere a la comprensión de la atención que debe ser dada por aquellos que investigan el desarrollo político. De esta forma, se comprende como Hagopian (2000), que tal investigación debe enfatizar principalmente el desarrollo de los padrones de asociación política, de los canales de participación política, de las instituciones formales e informales de la representación política y la gobernanza.

La segunda se refiere a la definición de la democracia. En este sentido, se asume un concepto más extenso que aquel propuesto por toda la tradición oriunda de Schumpeter $(1961)^{6}$. Por consiguiente, es necesario para contrarrestar a la mera democracia electoral, un concepto que incluya otras dimensiones más allá del simple proceso de selección de los gobernantes. Por lo tanto, la elección es causa necesaria, pero no suficiente de una democracia.

A partir de una concepción de la democracia que tiene en cuenta cinco dimensiones, el Democracy Index ${ }^{7}$ incluye no solo aspectos institucionales, sino también de comportamiento y culturales. Las instituciones son importantes porque moldan el comportamiento

3 Cf. Hagopian e Mainwaring (2005).

4 Cf. Hagopian (2000)

5 Cf. Albuquerque (2009).

6 Además de las definiciones de procedimiento minimalistas de Schumpeter, la definición de la democracia adoptada aquí también se distingue de las definiciones no procedimentales, a ejemplo de Bollen (1980) y (1991).

7 Se señala que no existe una preocupación de los diversos índices, incluyendo el Democracy Index, en considerar las relaciones entre civiles y militares y, por tanto, el control civil democrático sobre las fuerzas armadas como una dimensión importante de la democracia. Latinoamérica vio en los últimos sesenta ańos 36 golpes de estado, la mayor parte de los cuales se produjeron con la participación de las élites civiles y militares. Para que no se diga que estos golpes se produjeron como consecuencia de la coyuntura política de la Guerra Fría, se registran aquí los ocurridos después de este período: Haiti (1991 e 2004), Peru (1992), Venezuela (2002), Equador (2005) e Honduras (2009) (Andréia Passos et alii 2007) 
de los actores sociales. Sin embargo, son igualmente importantes los roles que cumplen la agencia y los valores en la creación, el mantenimiento y el cambio institucional.

La problemática central de este trabajo radica en la razón de la mala calidad y la no consolidación de la mayor parte de las democracias latinoamericanas. En un intento de encontrar una respuesta, se afirma, conjeturalmente, que estas democracias carecen de su dimensión liberal.

El argumento utilizado es que los regímenes políticos latinoamericanos han dejado a un lado las dimensiones de una democracia plena o liberal, como el buen funcionamiento de las instituciones democráticas y del Estado de Derecho, la significativa participación de los ciudadanos en las deliberaciones de la comunidad política y el alto nivel de cultura política democrática requerido por una democracia sólida.

Por lo tanto, el principal objetivo de este estudio es proporcionar una explicación plausible a la luz de la evidencia empírica, a su hipótesis principal: la ausencia de la dimensión liberal en las democracias latinoamericanas.

Sirvieron como fuentes de datos para este análisis los Indexes of Democracy de 2006, 2008, 2010 y 2011. Una parte de los datos se toman directamente de los Indexes y se utilizaron en el análisis. Otra parte se procesó y solo entonces fue analizada.

Para lograr el objetivo propuesto, el texto se divide fundamentalmente en cuatro partes: la primera (sección dos) aborda la definición y mensuración de la democracia presentada por el Democracy Index Se explican las dimensiones de la democracia, la tipología de los regímenes políticos y la puntuación que los clasifica en una de cuatro formas del régimen político.

La segunda parte (sección 3) trata de la mensuración y clasificación de los regímenes políticos en América Latina a partir del análisis de las puntuaciones de la MEs (media de las puntuaciones de cada dimensión por país) de cada dimensión de la democracia. Se divide en dos subsecciones: la primera presenta los procedimientos utilizados y la segunda los realiza.

La tercera parte (Sección 4) establece una comparación entre las dimensiones de la democracia a partir de la media de las puntuaciones que señalan sus capacidades y deficiencias.

Por último, la cuarta y última parte (sección cinco), hace un análisis y clasificación de los regímenes políticos tomando como base la media de la puntuación total.

\section{Democracy Index: Definiendo y Mensurando la Democracia}

El Democracy Index es una producción de Economist Intelligence Unit cuyo principal objetivo es proporcionar una definición y metodología empírica para medir el régimen 
democrático y la clasificación de los regímenes políticos en 167 países. Ofrece una definición empírica de la democracia que va más allá que el mero hecho electoral y propone cinco dimensiones constitutivas de este régimen: a) el proceso electoral y pluralismo; b) el funcionamiento del gobierno c) la participación política; d) la cultura política; y e) las libertades civiles. Su metodología ${ }^{8}$ propone una mensuración de este régimen en una escala de 0 a 10 basada en la evaluación de 60 indicadores agrupados en estas cinco dimensiones. También propone una tipología de regímenes políticos que comprenden la democracia plena, la democracia defectuosa o imperfecta, el régimen híbrido y el régimen autoritario.

En la dimensión relativa al proceso electoral y pluralismo (electoral process and pluralismo) se lleva a consideración, entre otros indicadores, la existencia de elecciones libres y justas para el Legislativo y el Ejecutivo, la existencia del sufragio universal para la población adulta, la igualdad de condiciones en la campańa electoral, las condiciones de posibilidad del elector votar sin amenazas significativas de los organismos estatales y no estatales, la aceptación de la transparencia de la financiación de los partidos políticos, la libertad de organización partidaria independiente del Estado, la posibilidad efectiva de los partidos de la oposición ganar el gobierno y la posibilidad que los ciudadanos formen organizaciones libres de la interferencia del Estado.

En cuanto al funcionamiento del gobierno (functioning of government) algunos de los indicadores considerados son: la importancia de los representantes elegidos libremente implementar las políticas del gobierno, la condición del Legislativo como órgano político supremo con clara hegemonía sobre los otros poderes del Estado, la existencia de un sistema eficaz de checks and balances en el gobierno, la inexistencia de poderío extranjero en la determinación de las funciones o políticas gubernamentales, la ausencia de interferencias indebidas en el gobierno por los militares y servicios de seguridad, la ausencia de ejercicio de poder paralelo significativo a través de grupos económicos, religiosos o de otra orden con las instituciones democráticas, los votantes deben tener mecanismos de accountability en su relación con el gobierno en los períodos entre elecciones, la autoridad del gobierno debe extenderse a todo el territorio del país, las acciones gubernamentales deben ser abiertas y transparentes permitiendo el acceso público a la información, la corrupción no debe ser un problema significativo, etc.

Con respecto a la participación política (political participation) aquí están algunos de los indicadores relevantes: un alto porcentaje de participación en las elecciones, la existencia de un grado razonable de autonomía y voz en los procesos políticos de las minorías (étnicas, religiosas, etc.), una considerable participación de las mujeres en el Parlamento, el compromiso ciudadano con la política, un elevado nivel de adultos alfabetizados (por

8 Cf. Index (2011), pp. 29-31. 
encima del 90\%) y un serio esfuerzo por parte de las autoridades para promover la participación política.

En lo relativo a la cultura política (political culture) son los principales indicadores: la existencia de un grado razonable de consenso y cohesión por parte de la sociedad para apoyar el funcionamiento de una democracia, un bajo porcentaje de la población que desee liderazgos fuertes sin parlamentos ni elecciones, un bajo porcentaje de la población que prefiera ser gobernado por militares y / o tecnócratas, alta proporción de la población (más del 80\%) creer que la democracia beneficia al desempeńo económico, un alto grado de apoyo a la democracia (por encima de 90\%) y una fuerte separación entre iglesia y estado.

Finalmente, en cuanto a las libertades civiles (civil liberties) se tienen en cuenta los siguientes indicadores, entre otros: la existencia de la libertad de los medios electrónicos, la existencia de medios de comunicación robustos, la libertad de expresión y la protesta, la libertad de organización profesional y sindical, la ausencia de la tortura por parte del Estado, el grado de independencia del poder judicial en relación a la interferencia gubernamental, el grado de libertad y tolerancia religiosa, la igualdad judicial, un sistema eficaz de seguridad pública, la protección de los derechos de propiedad y la libertad empresarial, la protección de las libertades civiles, la ausencia de discriminación significativa de raza, color, credo, etc.

Sobre la base de estas categorías se clasifican los regímenes políticos a partir de ciertos valores mensurados en una escala de 0 a 10 . Así, para que un régimen político sea considerado una democracia plena (full democracy), es necesario para se obtenga una puntuación total (overall score) de 8 a 10. Para que sea clasificada como una democracia imperfecta (flawed democracy), se hace necesario obtener una puntuación total de 6-7,9; Cuando un régimen político tiene una puntuación total de 4-5,9; recibe la clasificación híbrido (bybrid regime). Por último, cuando un régimen obtiene puntuación por debajo de cuatro se considera autoritario (authoritarian regime).

De acuerdo con el Democracy Index, la plena democracia se caracteriza por poseer: a) el respeto de las libertades políticas y civiles; b) una cultura política democrática; c) una gobernanza satisfactoria; d) un poder judicial independiente cuyas decisiones surtan efecto; y e) los problemas en el funcionamiento de la democracia son limitados.

Las democracias imperfectas incluyen: a) respeto a las libertades políticas y civiles; b) problemas de gobernanza; c) los bajos niveles de participación política; y d) una cultura política subdesarrollada.

Los regimenes híbridos poseen: a) elecciones con irregularidades sustanciales que a menudo les impiden ser libres y justas; b) las deficiencias en el funcionamiento del gobierno, la participación y cultura política (mayores que las democracias imperfectas); c) la 
corrupción generalizada; d) frágil Estado de derecho; e) sociedad civil poco desarrollada;

e) fuerte amenaza a la libertad de prensa; y f) poder judicial sin independencia.

Por último, los regímenes autoritarios consideran: en muchos casos los regímenes literalmente dictatoriales; b) la ausencia de pluralismo político o una fuerte limitación del mismo; c) algunas instituciones formales de la democracia, pero con poca robustez; d) las elecciones, cuando existen, no son libres ni justas; e) una fuerte violación de las libertades civiles; f) solamente los medios estatales o controlados por grupos apoyan al régimen; g) la censura y la represión contra los críticos del régimen; h) absoluta falta de independencia del poder judicial.

\section{Mensurando y Clasificando los Regímenes Políticos por Dimensión}

Esta sección tiene como objetivo mensurar y clasificar, con base en el Democracy Index, los distintos regímenes políticos latinoamericanos. Se divide en dos subsecciones: la primera, establecen brevemente los procedimientos utilizados para la mensuración y clasificación de la democracia y otros sistemas; la segunda lleva a cabo tales procedimientos.

\subsection{Procedimientos Utilizados Basados en el Democracy Index}

La medición y clasificación de los regímenes políticos latinoamericanos basados en el Democracy Index (en adelante Index), siguieron los siguientes pasos:

a) Inicialmente, se seleccionaron 20 de los 24 países de América Latina y el Caribe constante de los Indexes, con exclusión de Guyana, Surinam, Jamaica y Trinidad y Tobago.

b) En segundo lugar, los resultados de las cinco dimensiones de la democracia fueron tomadas directamente de los índices.

c) En tercer lugar, se construyó una tabla llamada "Regímenes políticos en América Latina” con base en la puntuación de los Indexes que contienen los siguientes elementos: la media de las puntuaciones de cada dimensión por país (MEs), la media de las puntuaciones totales por país (METs) y la media de las puntuaciones totales por dimensión de la democracia (MTDi).

d) En cuarto lugar, fueron realizadas las mensuraciones de las dimensiones y clasificaciones de los regímenes políticos basados en las puntuaciones de los cuatro Indexes y sus promedios, de las METs y MEs de cada dimensión.

e) Por último, mantuvimos todos los demás contenidos de los Indexes relativos a la tipología de los regímenes políticos y los indicadores de su clasificación. 


\subsection{Mensurando y Clasificando los Regímenes Políticos por Dimensión}

Para realizar este procedimiento, serán levados en consideración las puntuaciones de los Indexes y las MEs relativas a las dimensiones que constituyen la definición de democracia adoptada.

\subsubsection{Proceso Electoral y Pluralismo}

El punto de partida de cualquier democracia consiste en el proceso electoral a través del cual los miembros del Ejecutivo y del Legislativo son seleccionados. Para que las elecciones sean consideradas democráticas se hace necesario que las mismas se realicen periódicamente, en un clima de libertad y de relativa igualdad de condiciones para los que de ellos participan. Se hace necesario todavía que la ciudadanía sea inclusiva? ${ }^{9}$ De esa forma, la garantía de las diversas libertades civiles, así como de los derechos políticos, son condiciones necesarias para considerar un proceso electoral plural, libre y justo. En Latinoamérica, con excepción de Cuba, la casi totalidad de los países contempla, en larga medida, estos requisitos.

Esta es la dimensión en la cual los países obtuvieron las mejores puntuaciones. Así, tanto del punto de vista de las puntuaciones como del punto de vista de su promedio, casi todos los países se posicionaron bien. La única excepción puede ser hecha a Cuba con puntuación 1,75 en todos los Indexes.

Señalando el Index de 2006 y 2008 se verifica que de los 20 países, 15 obtuvieron las puntuaciones compatibles con los de una democracia plena, 3 con los de una democracia imperfecta, 1 con los de un régimen híbrido y 1 con los de un régimen autoritario.

En el Index de 2010 se observa que 13 dos 20 países alcanzaron puntuaciones pertinentes a los de una democracia plena. Por lo tanto, dos a menos que en los Indexes de 2006 y 2008. Al contrario, el número de puntuaciones compatibles con los de una democracia imperfecta aumentó de 3 en los dos Indexes anteriores para 5. Por fin, se mantuvieron 1 en las puntuaciones pertinentes a los de un régimen híbrido y 1 en los de un régimen autoritario.

En el Index de 2011 el número de puntuaciones compatibles con los de una democracia plena decreció de 13 para 12, los relativos a los de una democracia imperfecta de 5 para 4 , los regímenes híbridos aumentaron de 1 para 2 y se mantuvo 1 en régimen autoritario.

La media total de las puntuaciones de esta dimensión (MTDi) es de 8,19. El gráfico 1 ilustra el número de regímenes políticos por Index y por la MEs.

9 Cf. Dahl (1971) 


\section{Regímenes por la Dimensión Proceso Electoral y Pluralismo}

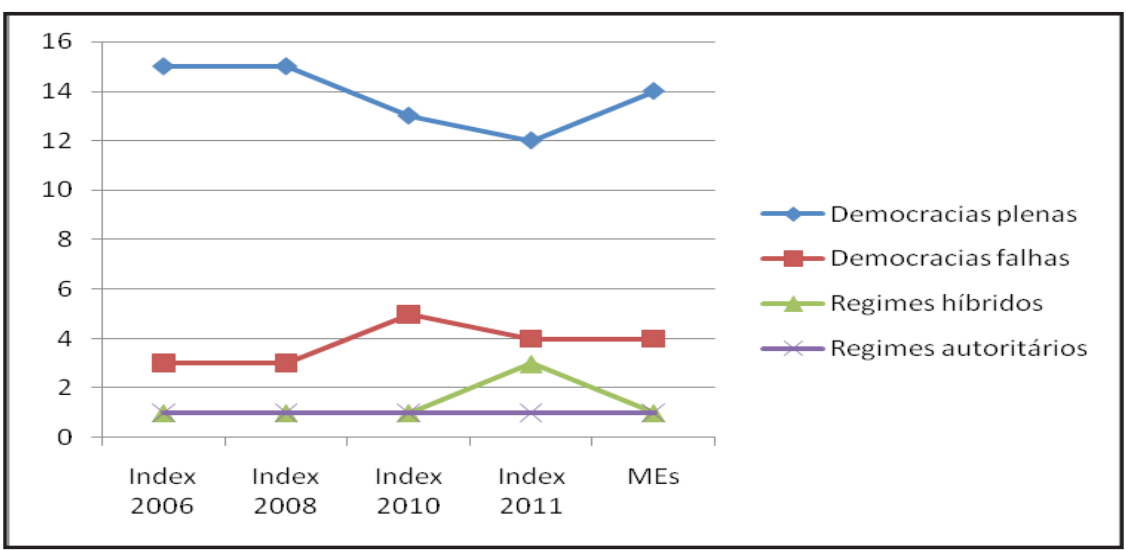

GRÁFICO 1

En síntesis, en lo que respecta a la categoría proceso electoral y pluralismo, los países de América Latina obtuvieron buenas puntuaciones y medias. Eso significa que en la mayor parte de los países no hay graves problemas en relación a las elecciones, en lo que respecta a su regularidad, su libertad y su idoneidad. De modo que por las MEs de esta dimensión, 14 países alcanzaron valores relacionados a las democracias plenas (Argentina, Brasil, Chile, Colombia, Costa Rica, El Salvador, Guatemala, Honduras, México Panamá, Paraguay, Perú, República Dominicana y Uruguay). Cuatro países obtuvieron las MEs pertinentes a las democracias imperfectas (Bolivia, Ecuador, Nicaragua y Venezuela). El Haití obtuvo media compatible con los regímenes híbridos y, por fin, Cuba, se mantuvo en el nivel de los países autoritarios. Ahora veamos la próxima dimensión.

\subsubsection{Funcionamiento del Gobierno}

Las elecciones para el Legislativo y el Ejecutivo no son suficientes para definir a un régimen democrático. Vale recordar que en el siglo pasado, en algunos casos, los líderes y los partidos totalitarios ascendieron al poder político a través de los mecanismos electorales. Por lo tanto, las elecciones son necesarias, no obstante insuficientes. De esta forma, es saludable que exista un sistema de accountability, tanto horizontal como vertical ${ }^{10}$, proporcionando la limitación de poder de los gobernantes elegidos y contribuya para la buena calidad de la democracia. Se necesita, por tanto, de medios que propicien tanto el control del Estado a través de sus mecanismos propios, como aquellos relacionados con la sociedad civil.

10 Cf. Guillermo O’Donnell (1998). 
En este sentido, el funcionamiento del gobierno en América Latina se ha configurado como uno de los primeros obstáculos a la consolidación de un régimen democrático. La primera señal de que esta es una dimensión problemática puede ser verificado en la fuerte caída de su MTDi, es decir, 5,88, en comparación con el anterior 8,19. Por tanto, es 2,31 menos que la media total del proceso electoral y pluralismo. Se pasa al análisis por Index.

En el Index de 2006 se observa que solo 3 de los 20 países alcanzaron un promedio compatible con las democracias plenas. Las democracias imperfectas fueron computadas en 5 países. Los regímenes híbridos alcanzan los números más altos hasta este momento, 9. Y, por último, 3 regímenes son clasificados como autoritarios.

En el Index de 2008 se mantiene el número de calificaciones correspondientes a una democracia plena, es decir, 3, los puntajes compatibles con las democracias imperfectas fueron alcanzados por 6 países, por lo tanto, uno más que en el Index anterior, los países que han logrado puntuaciones en el padrón de los regímenes híbridos aumentaron de 9 a 10 y solo un régimen autoritario se mantuvo.

En el Index de 2010 y 2011 se mantuvieron los mismos números: 3 puntuaciones en el nivel de democracias plenas, 7 en las democracias imperfectas, 8 en los regímenes híbridos y 2 en los regímenes autoritarios. Por lo tanto, ocurrió el aumento de 1 país con puntaje de democracia imperfecta, dos países disminuyeron sus puntuaciones de los regímenes híbridos y aumentó 1 país de los que recibieron estándar de gobierno autoritario.

El número de los regímenes políticos en relación a esta dimensión, por Index y por la MEs, se pueden ver en el gráfico 2 .

\section{Regímenes por la Dimensión Funcionamiento del Gobierno}

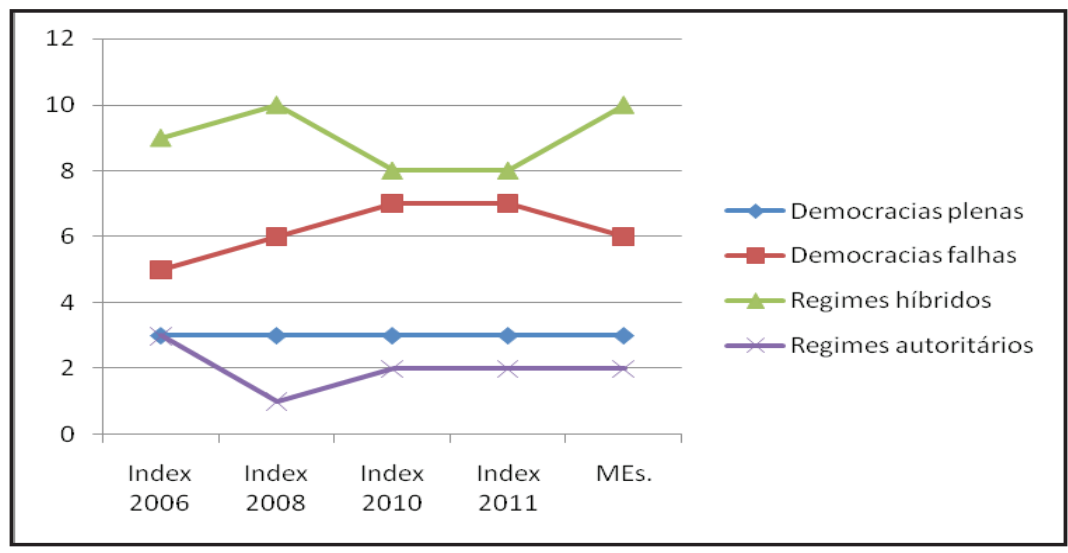

GRAFICO 2 
Se observa, por lo tanto, que en relación al funcionamiento del gobierno las MEs sufren en general, una disminución considerable. En cuanto a las MEs de los 20 países, solo tres obtuvieron medias compatibles con una democracia plena (Uruguay, Chile y Costa Rica). Cinco países lograron medias que los clasifican como democracias imperfectas (Brasil, Panamá, México, Guatemala y Colombia). Diez países obtuvieron medias de regímenes híbridos (Argentina, Bolivia, Cuba, El Salvador, Ecuador, Honduras, Nicaragua, Paraguay, Perú y República Dominicana). Por último, dos países tuvieron las más bajas MEs, Haití y Venezuela.

Así, por medio de las puntuaciones se infiere que a pesar de elecciones periódicas, libres y relativamente justas, el funcionamiento del gobierno deja mucho que desear. Una de las principales causas que se puede destacar es la ausencia de un sistema efectivo de accountability. Esta ausencia permite que después de las elecciones los gobernantes concentren en sus manos una amplia margen de poder político sobreponiéndose a los demás poderes y a la propia sociedad civil.

\subsubsection{Participación Política}

Una democracia requiere mucho más que un conjunto de instituciones. Sin embargo, las instituciones son importantes porque forman incentivos y expectativas que guían el comportamiento de los actores sociales, recompensándolos o castigándolos (Maravall y Przeworski 2003). No obstante, las instituciones no pueden prescindir de la agencia, ya que esto también tiene un impacto en la creación, el mantenimiento y el cambio institucional. Por lo tanto, la pasividad o la apatía de los actores sociales es incompatible con un régimen democrático. En América Latina la participación política es una de las dimensiones más problemáticas de la democracia, ya que exige a los ciudadanos un elevado nivel de cultura cívica $^{11}$. Los puntajes de las MEs de la participación política pasan a ser analizadas.

Debe decirse desde el principio que ningún país obtuvo una puntuación compatible con una democracia plena en cualquiera de los Indexes y, obviamente, en sus promedios. Peor que esto, en el Index de 2006 y 2008, solo Costa Rica logro puntuación en los valores de una democracia imperfecta. La mayor parte de los países (13) alcanzaron puntuaciones de regímenes híbridos y 6 de regímenes autoritarios.

En el Index de 2010, una vez más, ninguna puntuación acorde con una democracia plena. Costa Rica y México obtuvieron puntajes de una democracia imperfecta. Los regímenes híbridos incluyen a 10 países y los autoritarios a 8 .

Por último, el Index de 2011 aparece con 3 regímenes con puntajes de democracias imperfectas, 8 con puntuación de regímenes híbridos y 9 con puntuación de regímenes autoritarios.

11 Cf. Almond e Verba (1989) e Putnam (1996). 
La MTDi de 4,61 es la más baja entre todas las dimensiones. Esta 3,58 por debajo del MTD $i$ del proceso electoral pluralismo. En el gráfico 3 se muestran los regímenes políticos por las MEs de la participación política.

\section{Regímenes por la Dimensión Participación Política}

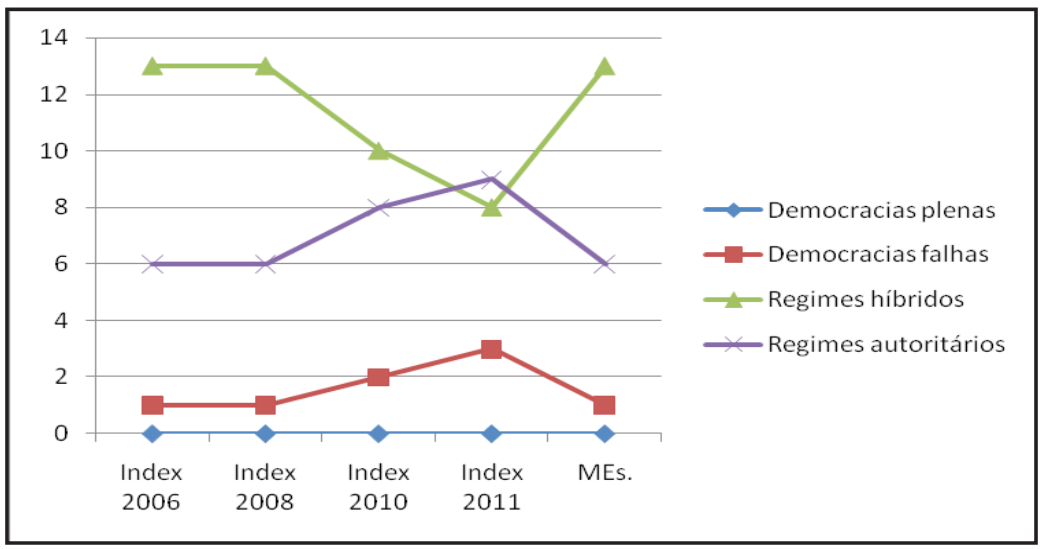

GRÁFICO 3

Se constata que en la dimensión participación política ningún país logró una puntuación compatible con una democracia plena. De los dos países considerados como tales, solo Costa Rica alcanzó en los cuatro Indexes una puntuación compatible con el de una democracia imperfecta, 6,11. Además, solo México (2010 y 2011) y Bolivia (2011) lograron la misma puntuación. Panamá, Argentina y Venezuela obtuvieron 5,56, Paraguay y Ecuador 5,00. Haití $(2,78)$ y Cuba $(3,89)$ mantienen las mismas puntuaciones en todos los Indexes. Uruguay y Chile, dos de las tres mejores puntuaciones totales en todos los Indexes, decayeron a puntajes compatibles con regímenes híbridos.

Al observar las MEs de esta dimensión, se obtiene el peor resultado en todos los casos: no hay ninguna media compatible con una democracia plena. Solo un promedio se encuadra entre las de una democracia imperfecta. La media de 13 países fueron consistentes con los regímenes híbridos (Argentina, Bolivia, Brasil, Chile, Colombia, Ecuador, Honduras, México, Panamá, Paraguay, Perú, Uruguay y Venezuela). Por último, seis países obtuvieron el promedio equivalente a regímenes autoritarios: Cuba, El Salvador, Guatemala, Haití, Nicaragua y la República Dominicana. Finalmente, se registra que esta dimensión fue aquella en la que la mayor parte de los países están con puntajes compatibles con los regímenes autoritarios y que estas cifras están incrementando de 6 en (2006 y 2008) a 8 en (2010) y para 9 en (2011). 


\subsubsection{Cultura Política}

Análogamente a la participación política, la cultura política desempeña un papel vital en la calidad de un régimen democrático. No son pocos los países de América Latina donde las instituciones democráticas están mal evaluadas en relación con las instituciones coercitivas, especialmente las fuerzas armadas ${ }^{12}$. Por lo tanto, la creencia en las instituciones políticas democráticas es parte constituyente fundamental del proceso de consolidación de la democracia. Para esta dimensión, la observación inicial realizada de la participación política es aplicable, que ninguno de los países en cuestión obtuvo un promedio o incluso una sola puntuación, compatible con una democracia plena.

El Index de 2006 señala la falta de resultados en el nivel de democracias plenas. Solo 3 países tienen puntuaciones similares a las democracias imperfectas, 13 permanecieron entre los de regímenes híbridos y 4 a los regímenes autoritarios. La puntuación media fue de 4,88 .

El Índice de 2008 repite la situación en relación con los dos primeros regímenes y sufre un pequeño cambio entre los regímenes híbridos, que aumentó de 13 a 14 y entre los regímenes autoritarios decreció de 4 a 3. La MEDi es 4,97. A pesar de haber mejorado en relación a la participación política, la MTD $i$ es 3,22 más baja que la MTD $i$ proceso electoral y pluralismo.

Los Indexes de 2010 y 2011 muestran cifras idénticas: 5 países con puntajes consistentes con democracias imperfectas, 11 con puntajes similares a los regímenes híbridos y 4 con puntajes compatibles con los regímenes autoritarios.

Se observa en el gráfico 4 el número de regímenes políticos en relación a esta dimensión, por Index y por la MEs.

\section{Regímenes por la Dimensión Cultura Política}

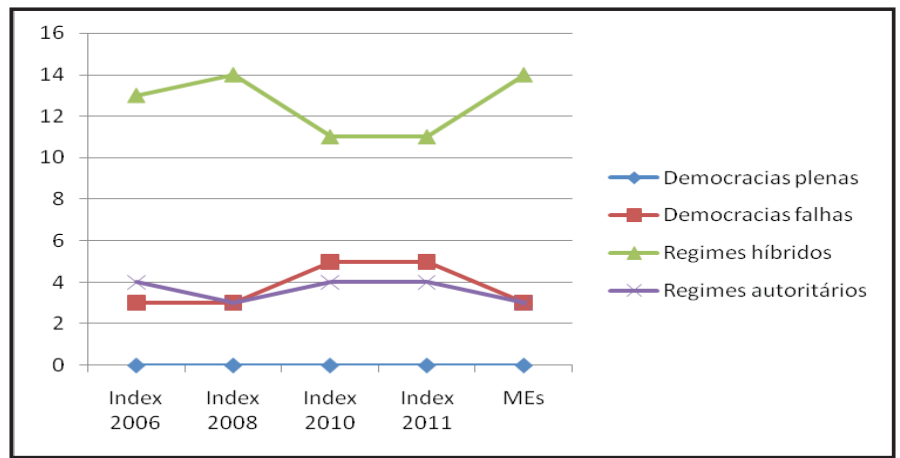

GRÁFICO 4

12 Cf. "A imagem das instituições públicas brasileiras", AMB, 2007 
Analizando la media de estas puntuaciones se puede notar que no son tan bajos como los pertinentes a la participación política, también permanecen en niveles mucho más bajos a los relacionados con el proceso electoral y pluralismo. Es, para ser más exactos, el segundo peor MTD $i$, es decir, 4,97.

De manera similar a la participación política, no surge una sola media ni puntuación que se encuentre dentro de los parámetros de democracias plenas. En el nivel de puntuación de las democracias imperfectas se clasifican solo tres países: Chile, Costa Rica y Uruguay. La mayor parte de la media, una vez más, inciden en el padrón de los regímenes híbridos en 14 países: Argentina, Brasil, Colombia, Cuba, El Salvador, Guatemala, Honduras, México, Nicaragua, Panamá, Paraguay, Perú, República Dominicana y Venezuela. Por último, la media de Bolivia, Ecuador y Haití se mantuvieron en el padrón de puntuación de los regímenes autoritarios.

\subsubsection{Liberdades Civis}

Las libertades civiles son derechos relacionados con las personas, tales como la libertad de conciencia, de expresión, de asociación y de reunión y otros. Evidentemente, estas libertades son de suma importancia para el establecimiento de una democracia, a pesar de que no sea más que electoral. Sin embargo, una vez que pasadas las elecciones, el grado de mantenimiento de estas libertades fijará el tono no solo del gobierno, sino de la propia democracia. En algunos países de América Latina, los gobiernos que surgen de los procesos electorales, a menudo, suprimen algunas de estas libertades civiles, lo que compromete la calidad de la democracia ${ }^{13}$.

Aunque los Indexes en cuestión no aborden explícitamente el requisito de un Estado de derecho que propicie una democracia liberal, está implícito en sus indicadores, cuando solicitan algunas protecciones de las libertades civiles y políticas, como la igualdad jurídica, que sigue siendo la piedra angular del Estado de derecho. Por consiguiente, esta dimensión de la democracia es de suma importancia.

Los Indexes de 2006 y 2008 muestran que 11 países tienen puntajes correspondientes a una democracia plena. Los que obtuvieron puntajes de una democracia imperfecta fueron 7. Los regímenes híbridos y autoritarios obtuvieron un puntaje para cada uno.

Asimismo, los Indexes de 2010 y 2009 muestran cifras similares: 10 países con puntajes compatibles con las de una democracia plena, con ocho puntuaciones referentes a las democracias imperfectas y 2 con puntajes en los padrones de los regímenes híbridos y autoritarios (nuevamente Venezuela y Cuba, respectivamente).

13 Cf. Hagopian y Mainwaring (2005). 
El MTD $i$ es de 7,87, por lo tanto, se mantiene por debajo de la dimensión relativa al proceso electoral y al pluralismo, es decir, 0,32. El gráfico 5 muestra el número de regímenes políticos con relación a esta dimensión.

\section{Regímenes por la Dimensión Libertades Civiles}

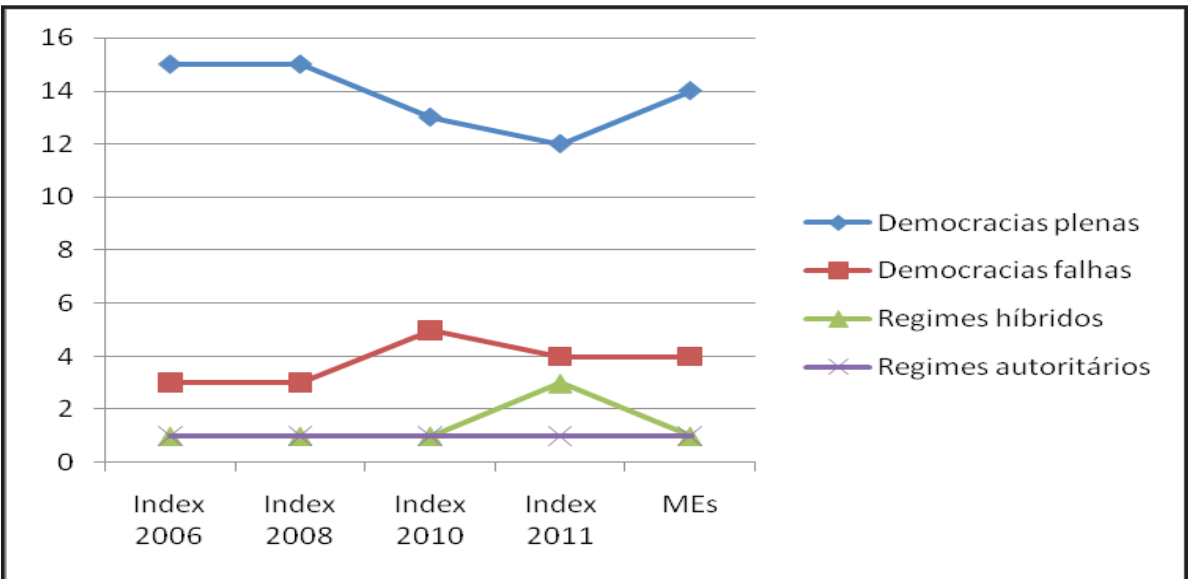

GRÁFICO 5

A diferencia de las MEs de las tres últimas dimensiones, las MEs de las libertades civiles muestran un escenario prometedor para la democracia, en particular en las esferas de las libertades civiles citadas anteriormente. Sin embargo, no se puede decir lo mismo en lo que respecta al grado de independencia judicial del gobierno, de un sistema eficaz de seguridad pública, de la ausencia de la tortura perpetrada incluso, por los organismos de seguridad del Estado. Estos son indicadores centrados también por los Indexes en relación con las libertades civiles.

De todos modos, al lado del proceso electoral dimensión y el pluralismo, aquí se obtuvieron los mejores resultados. Es el segundo mejor MTD $i$ de las cinco dimensiones, es decir, 7,87. De los 20 países, 12 lograron media en los niveles de una democracia plena (Argentina, Brasil, Chile, Colombia, Costa Rica, El Salvador, México, Panamá, Paraguay, Perú, República Dominicana y Uruguay). Otros seis obtuvieron media concerniente con las democracias imperfectas (Bolivia, Ecuador, Guatemala, Honduras y Nicaragua). Solo Venezuela y Cuba tuvieron promedios para los regímenes híbridos y autoritarios, respectivamente. El gráfico 6 muestra el número de regímenes gubernamentales por dimensión. 


\section{Las Cinco Dimensiones de la Democracia Comparadas}

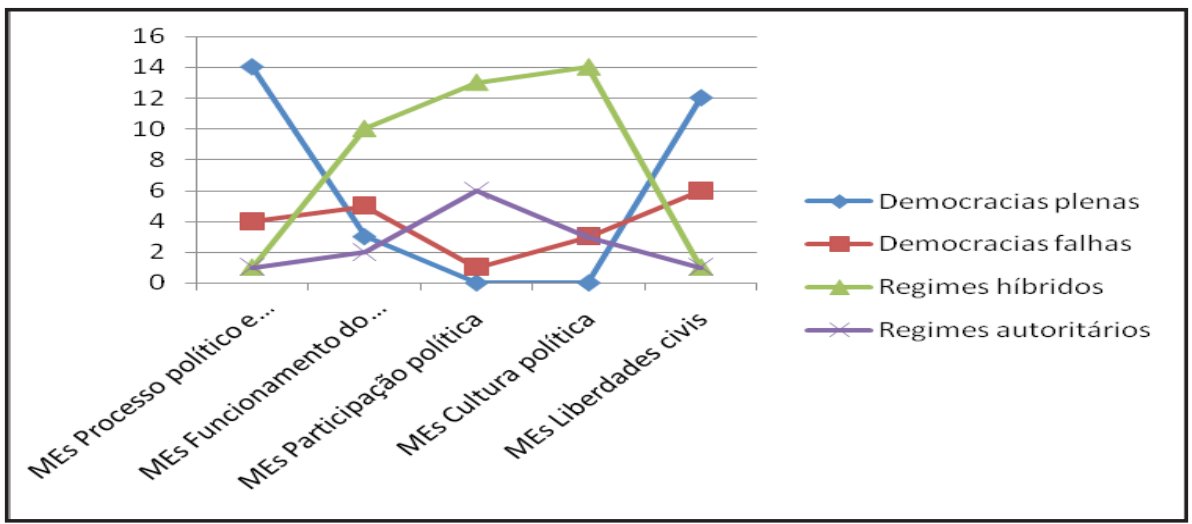

GRÁFICO 6

\section{Comparando las Cinco Dimensiones de la Democracia}

Cuando comparamos las dimensiones de la democracia a partir de la media de las puntuaciones de cada dimensión alcanzada por los países, señala claramente la diferencia entre dos buenos promedios, en relación con el proceso político y el pluralismo y las libertades civiles, un promedio que se puede considerar cerca de lo razonable, en relación con el funcionamiento del gobierno, y sobre todo los bajos promedios de participación y cultura política. El gráfico 7 ilustra bien esta situación.

\section{Las Cinco Dimensiones de la Democracia Comparadas}

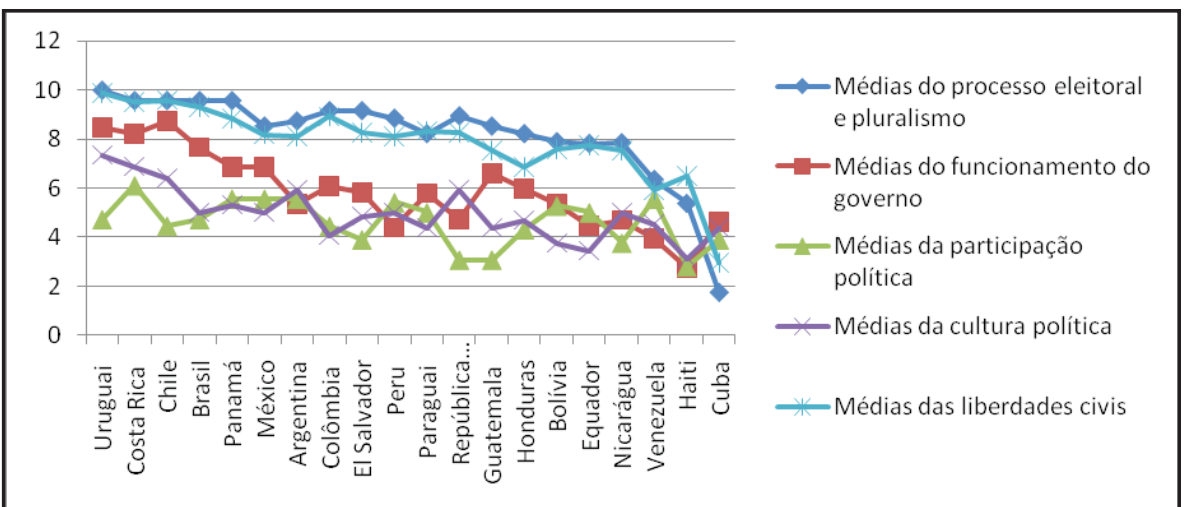

GRÁFICO 7 
Lo que se puede verificar es que las democracias latinoamericanas contienen, en gran medida, las propiedades constitutivas de una democracia electoral y las libertades civiles, en particular, las necesarias para la celebración de elecciones periódicas, libres y justas. La mayor parte de los países en cuestión tienen promedios entre excelente y buena en las dimensiones del proceso electoral y pluralismo (MTD $i=8,19)$ y las libertades civiles (MTD $i=7,87)$. Aunque Haití tiene problemas con la media, se hace necesario tener en cuenta la situación excepcional que desde 2004 el país ha enfrentado en la restauración del orden político. Cuba, por supuesto, es un outlier.

En cuanto al funcionamiento del gobierno hubo una caída significativa de su promedio y de las dimensiones anteriores $(\mathrm{MTD} i=5,88)$. Es posible enumerar algunos de los principales problemas de los países latinoamericanos en relación a esta dimensión. Entre ellos se encuentran: la hegemonía del poder ejecutivo sobre los otros poderes y, por tanto, la ausencia de un sistema eficaz de checks and balances; la ausencia de un sistema efectivo de accountability (que propicia la falta de transparencia de las acciones del gobierno y, como consecuencia, un alto grado de corrupción ${ }^{14}$ ); la ausencia de un efectivo control civil democrático sobre las fuerzas armadas, de inteligencia y de seguridad, en vista de la naturaleza del carácter pretoriano de las sociedades latinoamericanas ${ }^{15}$. Esto requiere de una atención especial teniendo en cuenta que la falta de control de las autoridades civiles sobre las instituciones coercitivas ha contribuido fuertemente a las graves violaciones de los derechos humanos ${ }^{16}$.

Pero es en la dimensión de la participación política que los problemas se presentan de forma más acentuada $(\mathrm{MTD} i=4,60)$. Es interesante notar que en América Latina el voto permanece obligatorio en 13 países (Argentina, Bolivia, Brasil Chile, Costa Rica, Guatemala, Honduras, México, Paraguay, Perú, Uruguay y Venezuela) de 24 en todo el mundo ${ }^{17}$. Se constata que el efecto que confiere esta seudoparticipación en el ejercicio de la ciudadanía es demasiado perjudicial. Hay una baja participación de la mujer en la vida política, tanto en el Poder Ejecutivo como en el Legislativo. Hay poco compromiso de los ciudadanos con la política y escasos esfuerzos de las autoridades para promover la participación política. Por último, en algunos países hay un alto número de adultos analfabetos $^{18}$, lo que contribuye fuertemente a una mala calidad de la ciudadanía. Según

14 Ver indicadores del Corruption Perceptions Index 2011

15 Pocos aspectos de la modernización política son más impresionantes y comunes que la intervención de los militares en la política. juntas y golpes, revueltas y regímenes militares han sido un fenómeno constante en las sociedades latinoamericanas "Huntington (1975

16 Ver indicadores del Human Rights Watch

17 Cf. Soares (2004

18 Ver indicadores en el Anuario Estadístico de América Latina y El Caribe 2010 (CEPAL / ECLAC). En este Brasil tiene la octava tasa más alta del analfabetismo entre los 28 países de la región, el 11,1\%. Está adelante únicamente de Jamaica (11,3\%), República Dominicana (14,5\%), El Salvador (18,9\%), Honduras (22,0\%), Guatemala (28,2\%), Nicaragua $(31,9 \%)$ y Haití $(45,2 \%)$. 
datos de 2007 de la Campaña Latinoamericana por el Derecho a la Educación (CLADE), América Latina cuenta con 35 millones de analfabetos, la mayoría de estos 14 millones están en Brasil, es decir, aproximadamente un tercio de analfabetos en la región.

Por último, con respecto a la cultura política, nuevamente la media es más baja y su MTD $i$ es 4,97, es el segundo promedio más bajo. Algunos de los problemas de esta dimensión pueden ayudar a explicar estos resultados. El primero sería la ausencia de un grado razonable de consenso y cohesión por parte de la sociedad que apoya el funcionamiento de una democracia ${ }^{19}$. Una parte considerable de la población que desea: a) un liderazgo fuerte y sin parlamento ni elecciones, b) ser gobernado por militares y / o tecnócratas; una baja adherencia de la población a la idea de que la democracia que la democracia beneficia al desempeño económico, bajo nivel de apoyo a la democracia y un rechazo de la separación entre iglesia y estado.

\section{Clasificando los Regímenes Políticos por las METs}

Finalmente, se realizó una clasificación de los regímenes políticos latinoamericanos basados en la media de las puntuaciones totales. Se observa, una vez más, que de los 20 países seleccionados solamente dos están clasificados por las puntuaciones totales de los indexes como democracias plenas: Uruguay y Costa Rica. Cuando vemos el número de METs, Uruguay aparece primero en la clasificación con 8,08 y Costa Rica ocupa el segundo lugar con 8,05.

Las 12 METs siguientes indican las tres democracias imperfectas del continente latinoamericano con puntajes totales entre y 7 y 8 . Por lo tanto, Chile, Brasil y Panamá aparecen en el tercer, cuarto y quinto lugares con METs de 7,75, 7,25 y 7,23, respectivamente.

México con 6,83, Argentina con 6,74, Colombia con 6,53, El Salvador con 6,39, Perú, con 6,35, Paraguay con 6,34, la República Dominicana, con 6,18, Guatemala con 6,02 y Honduras con 6,01 complementa este panorama. Sus posiciones en el ranking ocupan, respectivamente, del $6^{\circ}$ al $14^{\circ}$ lugares.

Bolivia con 5,79, Nicaragua con 5.76, Ecuador con 5,69, Venezuela con 5,26 y Haití con 4,1 ocupan del $15^{\circ}$ al $19^{\circ}$ colocaciones en el rango. Desde sus METs sus regímenes se clasifican como híbridos.

Por último, Cuba aparece como el único gobierno autoritario en este universo con el último lugar en el ranking de América Latina y con METs de apenas 3,52.

19 Ver indicadores del Latinobarômetro sobre "El apoyo a la democracia" (2011, pp. 26-29). 
Al clasificar los regímenes de gobierno por las METs de los cuatro Indexes, resulta que la mayoría de los países de América Latina se mantienen en la categoría de democracias imperfectas. El gráfico 8 ilustra esta situación.

\section{Regímenes por METs}

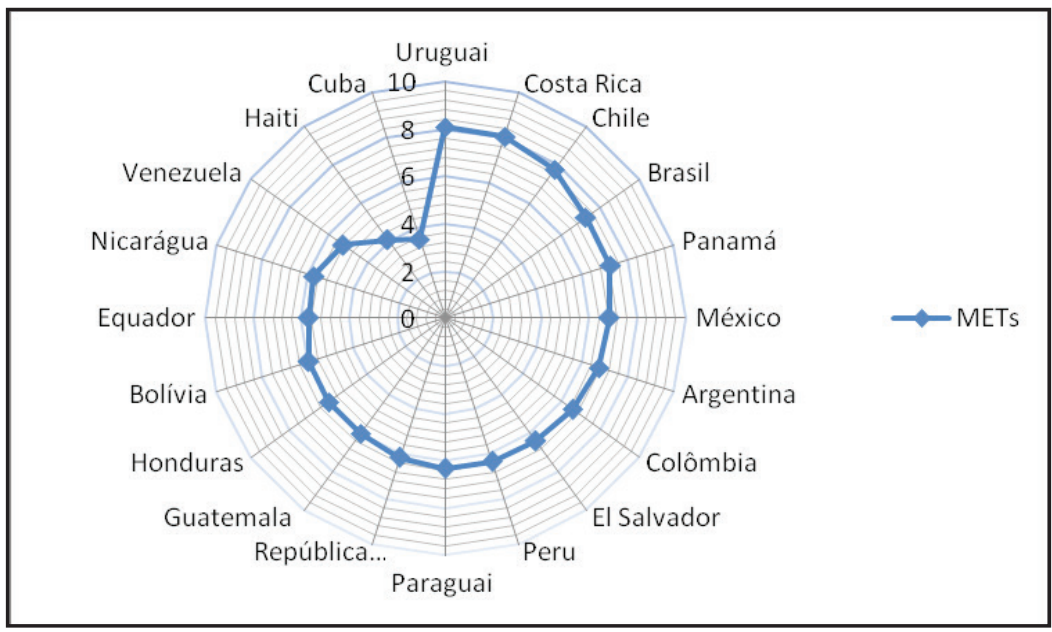

GRÁFICO 8

En resumen, por la METs en América Latina hay dos democracias plenas (10\%),12 democracias imperfectas (60\%), cinco regímenes híbridos (25\%) y un régimen autoritario $(5 \%)$. Por lo tanto, se puede afirmar, categóricamente, a la luz de las cifras presentadas, que las democracias imperfectas constituyen el universo más amplio de los regímenes políticos en América Latina.

\section{Conclusión}

Este trabajo trató de analizar las causas de las principales deficiencias de las democracias de América Latina que, en última instancia, impiden su consolidación. Por lo tanto, se utilizó uno de los Index de mayor reputación de la democracia, el Democracy Index. Además de los datos tomados directamente de sus versiones 2006, 2008, 2010 y 2011, fue construido una tabla (Regímenes políticos en América Latina) con un rango, la medias de las puntuaciones de las cinco dimensiones de la democracia (MEs), la media de sus puntuaciones totales (METs) y la media de las puntuaciones totales por dimensión (MTDi). A partir de ahí, hubo un análisis y clasificación de los regímenes de cada una de las dimensiones democráticas. Luego se hizo un análisis comparativo de las cinco 
dimensiones de la democracia basado en las MEs. Por fin, se analizaron la media para las puntuaciones totales y se elaboró una clasificación de los regímenes políticos tomándolas como base.

La hipótesis inicial de esta investigación es que la mayoría de las democracias latinoamericanas son meramente electorales o con denominaciones diversas que difieren de una democracia liberal. Su principal argumento es que hay negligencia en la región con respecto a las dimensiones que extrapolen las elecciones y de algunas libertades civiles que son condiciones necesarias. De esta forma, todas las demás dimensiones de la democracia, como el funcionamiento del gobierno, participación y cultura política son relegadas a un segundo plano.

El propósito principal de este trabajo fue tratar de explicar la mala calidad de la democracia latinoamericana y su no consolidación, señalando sus causas a la luz de la evidencia empírica presentadas. Se cree que a partir del análisis de los datos proporcionados por los Indexes y por la tabla de los Regímenes políticos Latinoamericanos, construida por los autores, se puede afirmar que este objetivo fue, en cierta medida, exitoso.

Al término de este trabajo es posible afirmar las siguientes conclusiones acerca de las dimensiones de las democracias de América Latina:

10) Ninguna de las democracias tiene problemas considerables en el proceso electoral y el pluralismo;

2o) Seis países (Argentina, El Salvador, Perú, Paraguay, República Dominicana y Honduras) tienen problemas razonables en cuanto a la dimensión funcionamiento del gobierno;

$3^{\circ}$ ) Con excepción de Costa Rica, todos los demás tienen graves problemas relacionados con la dimensión de la participación política, en particular los de $\mathrm{El}$ Salvador, República Dominicana y Guatemala;

$\left.4^{\circ}\right)$ Todos, excepto Uruguay, Costa Rica y Chile, tienen serios problemas con respecto a la cultura política;

5) Por último, ninguno de ellos tiene problemas con las libertades civiles.

No obstante estas fragilidades, las democracias latinoamericanas han logrado diferentes formas de consolidar la dimensión electoral, aunque no ha sido lo mismo con aquellas dimensiones pertenecientes a una democracia liberal. Estas tienen implicaciones más duraderas que una simple formalización de un gobierno democrático. Requieren de un sistema democrático en el que se contempla mucho más que el buen funcionamiento de las instituciones gubernamentales, la protección de las libertades políticas y civiles, la participación significativa de los ciudadanos en las deliberaciones sobre política y el alto nivel de la cultura política democrática. 
Al final de esta investigación, hay que confesar la fuerte propensión a predecir un futuro prometedor para América Latina desde el punto de vista de su desarrollo político, dado que, tiene sus raíces en 14 democracias de los 20 regímenes políticos analizados. Mejor que eso, solo uno está configurado como autoritario. Sin embargo y a pesar de esa inclinación, la prudencia impone una pregunta crucial e inevitable: ¿Qué camino tomaran las democracias imperfectas? ¿El de la consolidación? ¿La estancación? O el retroceso? Con la palabra, la historia.

\section{Referências}

A imagem das instituiçóes públicas brasileiras. AMB, Brasília, setembro de 2007. Disponível em: http://www.amb.com.br/docs/pesquisa/imagem_instituicoes.pdf. Acesso em: 12.01.2012.

Anuário Estadístico de América Latina y El Caribe 2010 (CEPAL/ECLAC). Disponívelem:http://websie.eclac.cl/anuario_estadistico/anuario_2010/docs/Introduccion_2010.pdf. Acesso em: 12.01.2012.

ALBUQUERQUE, Armando. Teoria democrática contemporânea: de Schumpeter a Mainwaring. In Marcelo Novelino e Agassiz Almeida Filho, Leituras Complementares de Direito Constitucional: Teoria do Estado. Salvador: JusPODIVM, 2009, pp. $131-151$.

ALMOND, G; VERBA, S. The civic culture revisited. Londres: Sage Publications, 1989.

BOLLEN, Kenneth A. Issues in the Comparative Measurement of Political Democracy in American Sociological Review, vol. 45, n. 3, 1980, pp. 370-390.

BOLLEN, Kenneth A. Liberal Democracy: Validity and Method Factors in Cross-National Measures in American Journal Political Science, vol. 37, n. 4, 1991, pp. 1207-1230.

CORPORACIÓN LATINOBARÓMETRO. Informe 2011. Disponível em: http:// www.latinobarometro.org/latino/latinobarometro.jsp. Acesso em 15 de fevereiro de 2012

COLLIER, David e LEVITSKY, Steven. Democracy with Adjectives: Conceptual Innovation in Comparative Research. World Politics Vol. 49, No. 3, Apr., 1997, pp. 430-451.

DIAMOND, Larry. Developing Democracy Toward Consolidation. Baltimore: The Johns Hopkins University Press, 1999.

HAGOPIAN, Frances. Political Development, Reviseted. Comparative Politics Studies, Vol. 33 No. 6/7, August/September 2000, 880-911. 
HAGOPIAN, Frances and MAINWARING, Scott P. The Third Wave of Democratization in Latin America: Advances and Setbacks. Cambridge: Cambridge University Press, 2005.

Human Rights Watch. Disponível em: http://www.hrw.org/. Acesso em: 12.01.2012.

HUNTINGTON, Samuel P. A ordem política nas sociedades em mudança. Tradução de Pinheiro de lemos. São Paulo: Editora Forense-Universitária e Editora da Universidade de São Paulo, 1975.

. (The Third Wave: Democratization in the Late Twentieth Century. Norman: University of Oklahoma Press, 1991.

MAINWARING, Scott. BRINKS, Daniel \& PÉREZ-LIÑÁN, Aníbal Classificando regimes políticos na América Latina. Revista de Ciências Sociais, Rio de Janeiro, vol. 44, n. 4, 2001, pp. 645 a 687.

MARAVAL, José María e PZREWOSRKI, Adam. Democracy and the Rule of Law. Cambridge: Cambridge University Press, 2003.

MARSHALL, T.H."Citizenship and Social Class", in T.H. Marshall, Class, Citizenship and Social Development. Chicago, The University of Chicago Press, 1964.

O’DONNELL, Guillermo. Accountability horizontal e novas poliarquias. Lua Nova: Revista de Cultura e Politica, São Paulo, CEDEC, n. 44, p. 27-103, 1998.

PASSOS, Andréia, MICHELSOHN, Davis e LIMA, José Antônio. “Os golpes de Estado na América Latina”. Revista Época. Disponível em: http://revistaepoca.globo.com/ Revista/Epoca/0,,EMI80186-15227,00.html. Acesso em: 81.01.2012.

SCHUMPETER, Joseph A. Capitalismo, socialismo e democracia. Rio de Janeiro: Fundo de Cultura, 1961.

SOARES, Paulo Henrique. Vantagens e desvantagens do voto obrigatório e do voto facultativo. (Consultoria Legislativa do Senado - Coordenação de Estudos). Textos para discussão 6. Brasília, 2004.

PUTNAM, Robert. Comunidade e democracia: a experiência da Itália moderna. Rio de Janeiro: FGV Editora, 1996.

ZAKARIA, Fareed. The Future of Freedom: Illiberal Democracy at Home and Abroad. New York e London: W. W. Norton \& Company, 2003.

ZAVERUCHA. Frágil democracia: Collor, Itamar, FHC e os Militares (1990-1998). Rio de Janeiro: Civilização Brasileira, 2000.

THE ECONOMIST INTELLIGENCE UNIT. Democracy Index 2006. http://www. economist.com/media/pdf/DEMOCRACY_INDEX_2007_v3.pdf. Acesso em 12.01.2012. 
THE ECONOMIST INTELLIGENCE UNIT. Democracy Index 2008. Disponível em: http://graphics.eiu.com/PDF/Democracy\%20Index\%202008.pdf. Acesso em 12.01.2012.

THE ECONOMIST INTELLIGENCE UNIT. Democracy Index 2010. Disponível em: http://graphics.eiu.com/PDF/Democracy_Index_2010_web.pdf. Acesso em: 12.01.2012.

THE ECONOMIST INTELLIGENCE UNIT. Democracy Index 2011. Disponível em: http://www.eiu.com/public/topical_report.aspx?campaignid=DemocracyIndex2011. Acesso em: 12.01.2012.

TRANSPARENCY INTERNATIONAL. Corruption Perceptions Index 2011. Disponível em: http://cpi.transparency.org/cpi2011/results/. Acesso em: 12.01.2012. 


\section{APENDICE}

\begin{tabular}{|c|c|c|c|c|c|c|c|c|c|c|c|}
\hline 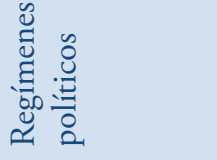 & $\hat{\bar{a}}$ & $\hat{\bar{\rho}}$ & $\overline{\boldsymbol{\rho}}$ & $\bar{\emptyset}$ & $\overline{0}$ & $\overline{0}$ & $\overline{\boldsymbol{a}}$ & $\overline{\boldsymbol{a}}$ & $\bar{\emptyset}$ & $\overline{\boldsymbol{\rho}}$ & $\overline{\boldsymbol{a}}$ \\
\hline 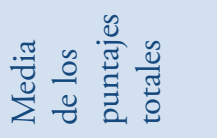 & $\begin{array}{l}\infty \\
0 \\
\infty \\
\infty\end{array}$ & $\begin{array}{l}n \\
0 \\
\infty\end{array}$ & $\stackrel{n}{n}$ & $\stackrel{\approx}{\curvearrowright}$ & $\stackrel{\sim}{N}$ & $\begin{array}{l}\infty \\
\infty \\
0 \\
0\end{array}$ & $\underset{\sigma}{\stackrel{+}{*}}$ & $\tilde{n}^{+1}$ & ڤે & $\hat{n}$ & 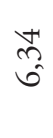 \\
\hline 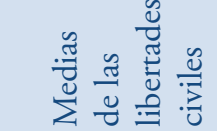 & $\begin{array}{l}\infty \\
\infty \\
\curvearrowleft\end{array}$ & 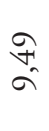 & $\stackrel{\curvearrowright}{\curvearrowleft}$ & $\hat{~}$ & $\begin{array}{l}\infty \\
\infty \\
\infty\end{array}$ & $\underset{\infty}{\stackrel{0}{\infty}}$ & $\begin{array}{c}\stackrel{\partial}{0} \\
\infty\end{array}$ & $\hat{\infty}$ & $\underset{\infty}{\stackrel{H}{N}}$ & $\begin{array}{l}\text { oे } \\
\infty\end{array}$ & $\vec{n}$ \\
\hline 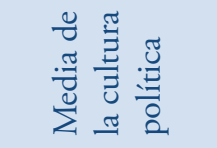 & $\tilde{n}$ & $\begin{array}{l}\infty \\
\infty \\
0 \\
0\end{array}$ & F & $\begin{array}{l}5 \\
\text { n }\end{array}$ & กี & $n$ & $\begin{array}{l}\vec{r} \\
\tilde{n}\end{array}$ & $\hat{O}$ & $\begin{array}{l}\mathscr{n} \\
\infty \\
\forall i\end{array}$ & $n$ & $\begin{array}{l}\infty \\
m^{2} \\
\forall\end{array}$ \\
\hline 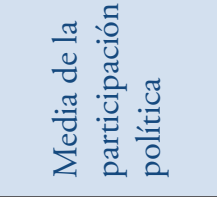 & $\underset{\mathbb{N}}{\stackrel{N}{*}}$ & $\bar{\sigma}$ & $\stackrel{i n}{\forall}$ & $\stackrel{\mathbb{N}}{\stackrel{*}{f}}$ & $\begin{array}{l}\stackrel{\sim}{n} \\
\stackrel{n}{*}\end{array}$ & 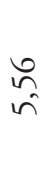 & $\begin{array}{l}\stackrel{2}{\sim} \\
\tilde{n}\end{array}$ & $\begin{array}{l}\stackrel{n}{f} \\
\forall\end{array}$ & $\begin{array}{l}\curvearrowright \\
\infty \\
\hat{n}\end{array}$ & $\begin{array}{l}\stackrel{F}{f} \\
\text { in }\end{array}$ & $n$ \\
\hline 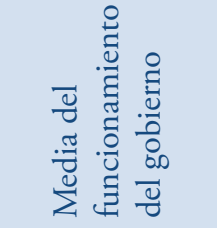 & $\begin{array}{l}\stackrel{\infty}{+} \\
\infty\end{array}$ & $\underset{\sim}{\stackrel{\sim}{ }}$ & $\stackrel{n}{\infty}$ & $\begin{array}{l}\infty \\
N\end{array}$ & $\begin{array}{l}\infty \\
\infty \\
6\end{array}$ & $\hat{\infty}$ & $\begin{array}{l}0 \\
\check{n} \\
\end{array}$ & $\begin{array}{l}\text { o } \\
\text { (0) }\end{array}$ & $\begin{array}{l}\infty \\
\infty \\
n\end{array}$ & $\stackrel{\text { ㅇ }}{\text { fi }}$ & $\begin{array}{l}\triangleright \\
\infty \\
\text { in }\end{array}$ \\
\hline 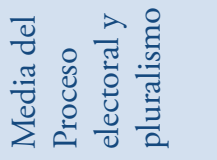 & $\begin{array}{l}0 \\
0 \\
0\end{array}$ & $\begin{array}{l}\infty \\
\\
a\end{array}$ & $\stackrel{\infty}{\sim}$ & $\begin{array}{l}\infty \\
\sim \\
\sim\end{array}$ & $\stackrel{\infty}{\sim}$ & $\stackrel{+}{\stackrel{\leftarrow}{\infty}}$ & $\stackrel{n}{\infty}$ & $\stackrel{\equiv}{a}$ & $\stackrel{ }{a}$ & $\begin{array}{l}\infty \\
\infty \\
\infty\end{array}$ & $\underset{\infty}{\infty}$ \\
\hline ᄋ & $\neg$ & $N$ & $n$ & $\psi$ & $n$ & $\bullet$ & $\Lambda$ & $\infty$ & $a$ & 으 & $\Xi$ \\
\hline चี & D & 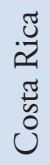 & $\frac{\mathscr{U}}{\mathscr{U}}$ & $\begin{array}{l}\overline{\text { त्र }} \\
\text { ص }\end{array}$ & 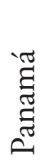 & 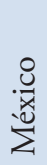 & 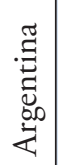 & $\frac{\pi}{\frac{\pi}{1}}$ & 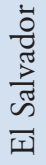 & $\overrightarrow{\vec{U}}$ & 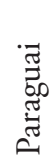 \\
\hline
\end{tabular}




\begin{tabular}{|c|c|c|c|c|c|c|c|c|c|c|c|}
\hline 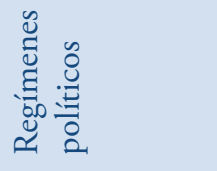 & $\bar{a}$ & $\overline{0}$ & $\overline{\mathrm{a}}$ & $\underset{I}{\mathbb{I}}$ & 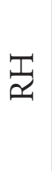 & 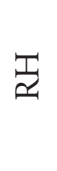 & $\underset{I}{I}$ & $\underset{I}{I}$ & 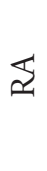 & & 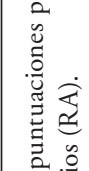 \\
\hline 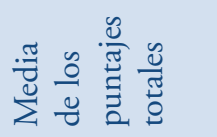 & $\stackrel{2}{\sigma}$ & 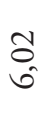 & రీ & $\hat{\tilde{n}}$ & $\begin{array}{l}\text { ఏ్ } \\
\text { « }\end{array}$ & $\stackrel{2}{\approx}$ & 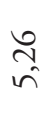 & $\begin{array}{l}\stackrel{0}{-1} \\
\underset{+}{*}\end{array}$ & $\tilde{n}$ & ஸે & 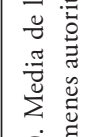 \\
\hline 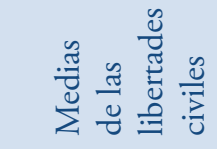 & $\underset{\infty}{\stackrel{+}{N}}$ & 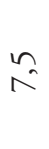 & 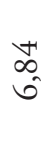 & $\hat{n}$ & $\stackrel{N}{\approx}$ & $\tilde{N}$ & $\begin{array}{l}\infty \\
\infty \\
\sim\end{array}$ & $\underset{\sigma}{\stackrel{F}{6}}$ & $\begin{array}{l}\text { ă } \\
\text { ते }\end{array}$ & $\begin{array}{l}\infty \\
N\end{array}$ & 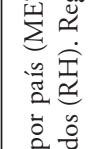 \\
\hline 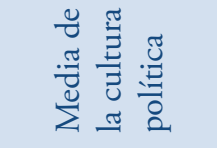 & $\begin{array}{l}\stackrel{+}{\hat{n}} \\
\hat{n}\end{array}$ & 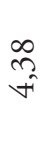 & Gे & $\stackrel{n}{n}$ & $\underset{r}{\stackrel{H}{*}}$ & $n$ & $\stackrel{⿱ ⺌}{\stackrel{5}{*}}$ & $\frac{m}{n}$ & \begin{tabular}{l}
$\infty$ \\
$\stackrel{n}{*}$ \\
\multirow{f}{*}{}
\end{tabular} & $\hat{\sigma}$ & 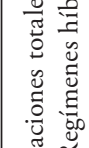 \\
\hline 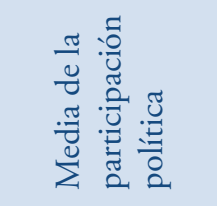 & $\begin{array}{l}\text { ¿ } \\
\text { n. }\end{array}$ & $\begin{array}{l}8 \\
\text { m }\end{array}$ & $\stackrel{2}{\forall}$ & $\stackrel{\infty}{\sim}$ & $n$ & $\frac{n}{n}$ & $\begin{array}{l}\stackrel{2}{n} \\
\sim\end{array}$ & $\frac{\infty}{\stackrel{\infty}{N}}$ & $\begin{array}{l}\curvearrowright \\
\infty \\
\text { ஸे }\end{array}$ & $\begin{array}{l}\vec{\sigma} \\
\forall\end{array}$ & 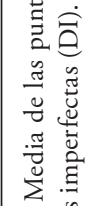 \\
\hline 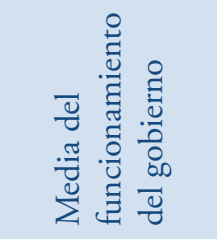 & $\stackrel{m}{\stackrel{m}{*}}$ & $\begin{array}{l}\vec{\sigma} \\
\sigma^{\prime}\end{array}$ & $\begin{array}{l}\stackrel{\infty}{\check{n}} \\
\tilde{n}\end{array}$ & $\begin{array}{l}\stackrel{0}{n} \\
\stackrel{n}{n}\end{array}$ & $\underset{f}{\stackrel{f}{f}}$ & $\underset{f i}{\stackrel{P}{r}}$ & $\begin{array}{l}\tilde{\varkappa} \\
\tilde{n}\end{array}$ & $\stackrel{n}{\hat{N}}$ & $\begin{array}{l}\stackrel{\leftarrow}{\sigma} \\
\forall\end{array}$ & $\begin{array}{l}\infty \\
\infty \\
n\end{array}$ & 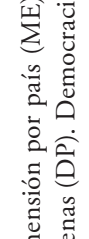 \\
\hline 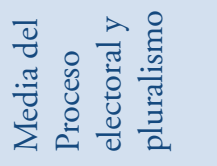 & $\begin{array}{l}\stackrel{2}{\circ} \\
\infty\end{array}$ & $\stackrel{+r}{\stackrel{+}{\infty}}$ & $\underset{\infty}{\infty}$ & ฉ̊ & $\stackrel{\infty}{\infty}$ & $\begin{array}{l}\infty \\
\infty \\
N\end{array}$ & 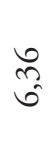 & $\begin{array}{l}\infty \\
\sim \\
\sim\end{array}$ & $\stackrel{n}{\approx}$ & $\stackrel{\curvearrowright}{\stackrel{\infty}{\infty}}$ & 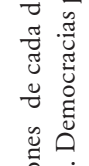 \\
\hline 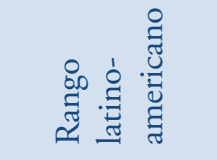 & $\stackrel{\sim}{\sim}$ & $\stackrel{n}{\sim}$ & $\Xi$ & $\cong$ & $\underset{-1}{0}$ & 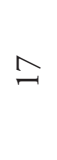 & $\stackrel{\infty}{-}$ & 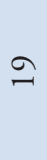 & 고 & & 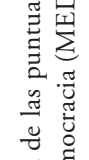 \\
\hline ॥ै & 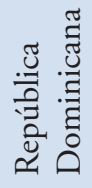 & 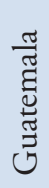 & 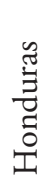 & 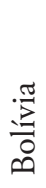 & 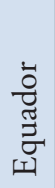 & 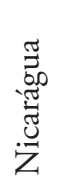 & 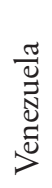 & : & $\frac{\pi}{3}$ & $\stackrel{\hat{\rho}}{\dot{\omega}}$ & 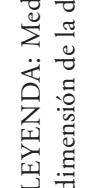 \\
\hline
\end{tabular}

